\title{
Elevation, azimuth, and polarization estimation with nested electromagnetic vector-sensor arrays via tensor modeling
}

\author{
Ming-Yang Cao ${ }^{1,2}$, Xingpeng Mao ${ }^{1,2^{*}}$ (D) and Lei Huang ${ }^{3}$
}

\author{
*Correspondence: mxp@hit.edu.cn \\ ${ }^{1}$ School of Electronics and \\ Information Engineering, Harbin \\ Institute of Technology, Xidazhi \\ Street, 150001 Harbin, China \\ ${ }^{2}$ Key Laboratory of Marine \\ Environmental Monitoring and \\ Information Processing, Ministry of \\ Industry and Information \\ Technology, Xidazhi Street, 150001 \\ Harbin, China \\ Full list of author information is \\ available at the end of the article
}

\begin{abstract}
In this paper, we address the joint estimation problem of elevation, azimuth, and polarization with nested array consists of complete six-component electromagnetic vector-sensors (EMVS). Taking advantage of the tensor permutation, we convert the sample covariance matrix of the receive data into a tensorial form which provides enhanced degree-of-freedom. Moreover, the parameter estimation issue with the proposed model boils down to a Vandermonde constraint Canonical Polyadic Decomposition problem. The structured least squares estimation of signal parameters via rotational invariance techniques is tailored for joint auto-pairing elevation, azimuth, and polarization estimation, ending up with a computational efficient method that avoids exhaustive searching over spatial and polarization region. Furthermore, the sufficient uniqueness analysis of our proposed approach is addressed, and the stochastic Cramér-Rao bound for underdetermined parameter estimation is derived. Simulation results are given to verify the effectiveness of the proposed method.
\end{abstract}

Keywords: Electromagnetic vector-sensor, Nested array, Parameter estimation, Tensor decomposition, Cramér-Rao bound (CRB)

\section{Introduction}

Electromagnetic vector-sensor (EMVS) has been widely used in a variety of applications such as localization, tracking, and beamforming [1-3]. A complete six-component EMVS contains spatially co-located three identical orthogonally electric dipoles and magnetic loops that could measure all six-components of the electromagnetic field [4, 5]. The model of EMVS was investigated in [6]. Different from scalar-sensor arrays, EMVS arrays that are composed of multiple EMVSs with particular configurations could detect both direction-of-arrival (DOA), i.e., elevation and azimuth, and polarization of incident sources. This polarization diversity brings us lots of advantages, such as resolving sources from the same DOA as long as they have different polarization states, providing better resolution ability, offering extra degrees-of-freedom (DOFs), and improving estimation performance [7-10].

(c) The Author(s). 2020 Open Access This article is licensed under a Creative Commons Attribution 4.0 International License, which permits use, sharing, adaptation, distribution and reproduction in any medium or format, as long as you give appropriate credit to the original author(s) and the source, provide a link to the Creative Commons licence, and indicate if changes were made. The images or other third party material in this article are included in the article's Creative Commons licence, unless indicated otherwise in a credit line to the material. If material is not included in the article's Creative Commons licence and your intended use is not permitted by statutory regulation or exceeds the permitted use, you will need to obtain permission directly from the copyright holder. To view a copy of this licence, visit http://creativecommons.org/licenses/by/4.0/. 
In order to utilize the aforementioned benefits, the DOA and polarization estimation with EMVS arrays could be cast as a multiple-parameter estimation problem which, however, turns out to be much more complicated than the scalar-sensor array case. Usually, they demand a time-consuming multi-dimensional searching procedure [11]. Also, the auto-pairing issue of different parameters cannot be neglected. Moreover, the steering matrix of an EMVS always has irregular structure, which aggravates the difficulty in the parameter estimation techniques. Several matrix-based DOA and polarization estimation methods have been proposed with the EMVS array. Most of them are the extensions of the existing DOA estimators with scalar-sensor arrays by taking into the physical structure of EMVSs into consideration. In [6], a vector cross-product approach to estimate the Poynting vector of the sources was presented. Eigenvector-based parameter estimators such as multiple signal classification (MUSIC) and estimation of signal parameters via rotational invariance techniques (ESPRIT) were developed in $[12,13]$, through utilizing either spatial or temporal invariance properties. These approaches show superior resolution abilities and estimation accuracy with tolerable computational burdens.

The multi-dimensional model, i.e., tensor model, was first linked with array processing under the framework with Canonical Polyadic Decomposition (CPD) in [14]. Interestingly, the tensor modeling techniques reverse the curse of multi-dimensional problems into blessing, which could achieve several benefits such as auto-paring of parameters, relaxed uniqueness condition, to name a few. Subsequently, tensor-based approaches were introduced to the EMVS array whose received data embodies multi-dimensional structure. In [15], the identifiability was analyzed with an EMVS array. More general CPD-based approach for EMVS array were developed in [16-19]. In parallel, higherorder singular value decomposition (HOSVD) which represents tensor-based low-rank approximation method, generalizes the concept of matrix-based SVD. HOSVD-based parameter estimators are able to provide higher estimation accuracy [20, 21]. The socalled tensor-MUSIC approaches were developed with the EMVS array in [22, 23], which require exhaustive multi-dimensional searching procedures.

Compared with uniform linear array (ULA), difference co-arrays such as nested and coprime arrays are able to provide more DOFs [24-26]. The nested array could construct virtual arrays that obtain as large as $\mathcal{O}\left(M^{2}\right)$ DOFs with $M$ physical sensors, which greatly improves the identifiability as well as resolvability and reduces mutual coupling effects between sensors. The stochastic Cramér-Rao bound (CRB) when the number of sources is more than the number of sensors was derived separately by [27-29]. It is worth noting that the aforementioned methods are only suitable for uncorrelated sources.

A nested array which is equipped with EMVSs was first developed by Han et al. in [30]. The nested EMVS array provides more DOFs and better resolvability than the EMVS ULA. However, the DOA and polarization vectors were not decoupled, meaning that the nested EMVS array requires a prior knowledge of the polarization state before performing MUSIC method to avoid exhaustive searching. A tensor model for nested EMVS array that could efficiently decouple the DOA and polarization was constructed in [31]. This method divides the nested EMVS array into several subarrays with respect to different polarization states. Then, it builds a tensor composed of multiple local covariances, followed by the CPD to obtain DOA and polarization estimations.

In this paper, we propose a novel methodology for tensor modeling and parameter estimation by means of utilizing the relationship between tensor permutation and the array 
structure. The proposed approach has several novelties and advantages: (1) A tensor model of nested EMVS array model is established through the property of tensor permutation, which is different from the complex produce proposed in [31]. (2) The proposed estimator is capable of achieving the auto-pairing of DOA and polarization state. (3) It is proved that the proposed method guarantees more DOFs through uniqueness condition analysis. (4) An underdetermined stochastic CRB for the nested EMVS array is derived as a benchmark for performance evaluation.

The remainder of this paper is organized as follows. Section 2 introduces the basic mathematical notations and constructs the tensor model of a nested EMVS array. In Section 3, we briefly review the Han's tensor modeling method and then propose our tensor modeling approach through multilinear algebra. In Section 4, we devise CPD and the structured least squares (SLS) ESPRIT method for joint DOA and polarization estimation. In Section 5, we analyze the sufficient uniqueness condition of the proposed method and derive the underdetermined stochastic CRB of the nested EMVS array. In Section 6, we give simulation results to demonstrate the effectiveness of our propose method. Section 7 concludes this work.

\section{Problem formulation}

\subsection{Notations}

Definition 1 (Tensor vectorization) $A$ tensor vectorization of an $\mathrm{N}$-dimensional tensor $\mathcal{A} \in \mathbb{C}^{I_{1} \times I_{2} \times \cdots \times I_{N}}$ is denoted as vec $(\mathcal{A}) \in \mathbb{C}_{n=1}^{N} I_{n} \times 1$ where vec $(\cdot)$ represents the vectorization operator.

Property 1 If an $N$-dimensional tensor $\mathcal{A} \in \mathcal{C}^{I_{1} \times \ldots \times I_{N}}$ could be expressed as the outerproduct of a sequence of vectors $\mathbf{a}_{n} \in \mathbb{C}^{I_{n} \times 1}, n=1,2, \ldots, N$, namely,

$$
\mathcal{A}=\mathbf{a}_{1} \circ \mathbf{a}_{2} \circ \cdots \circ \mathbf{a}_{N}
$$

where $\circ$ denotes the outer product, then, its tensor vectorization has the following structure as

$$
\operatorname{vec}(\mathcal{A})=\mathbf{a}_{N} \odot \mathbf{a}_{N-1} \odot \cdots \odot \mathbf{a}_{1}
$$

where $\odot$ represents the Khatri-Rao product.

Definition 2 (Tensor matrization) A matrix unfolding of an $\mathrm{N}$-dimensional tensor $\mathcal{A} \in$ $\mathbb{C}^{I_{1} \times I_{2} \times \cdots \times I_{N}}$ along n-mode is denoted as $\mathcal{A}_{(n)} \in \mathbb{C}^{I_{n} \times I_{n+1} \ldots I_{N} I_{1} \ldots I_{n-1}}$.

Definition 3 (The $n$-mode tensor-matrix product) The n-mode product of a tensor $\mathcal{A} \in$ $\mathbb{C}^{I_{1} \times I_{2} \times \cdots \times I_{N}}$ and a matrix $\mathbf{D} \in \mathbb{C}^{J_{n} \times I_{n}}$ along the $n$th mode is given by

$$
\begin{aligned}
& \mathcal{C} \triangleq \mathcal{A} \times{ }_{n} \mathbf{D} \\
& c_{i_{1}, i_{2}, \ldots, i_{n-1}, j_{n}, i_{n+1}, \ldots, i_{N}}=\sum_{i_{n}=1}^{I_{n}} a_{i_{1}, i_{2}, \ldots, i_{n}, \ldots, i_{N}} d_{j_{n}, i_{n}}
\end{aligned}
$$

where $\mathcal{C} \in \mathbb{C}^{I_{1} \times \ldots \times I_{n-1} \times J_{n} \times I_{n+1} \times \ldots \times I_{N}}$ and $\mathcal{C}_{(n)}=\mathbf{D} \mathcal{A}_{(n)}$. 


\subsection{Signal model}

For a six-component EMVS, it consists six spatially co-located antennas, i.e., three orthogonally electric dipoles and three orthogonally magnetic loops. We adopt $\mathbf{e}_{k} \triangleq$ $\left[e_{x_{k}}, e_{y_{k}}, e_{z_{k}}\right]^{T}$ and $\mathbf{h}_{k} \triangleq\left[h_{x_{k}}, h_{y_{k}}, h_{z_{k}}\right]^{T}$ to denote the $k$ th source's electromagnetic field characterized by electric and magnetic triads along $x-, y$-, and $z$-axis, respectively. The diagram of an EMVS under Cartesian coordinates is shown in Fig. 1.

We assume that there are no mutual coupling effects within each EMVS. Thus, the physical polarization vector of $k$ th source observed by an EMVS is a collection of $\mathbf{e}_{k}$ and $\mathbf{h}_{k}$

$$
\begin{aligned}
& \mathbf{p}_{k} \triangleq\left[e_{x_{k}} e_{y_{k}} e_{z_{k}} h_{x_{k}} h_{y_{k}} h_{z_{k}}\right]^{T} \\
& \triangleq\left[\begin{array}{cc}
\cos \phi_{k} \cos \theta_{k} & -\sin \phi_{k} \\
\sin \phi_{k} \cos \theta_{k} & \cos \phi_{k} \\
-\sin \theta_{k} & 0 \\
-\sin \phi_{k} & -\cos \phi_{k} \cos \theta_{k} \\
\cos \phi_{k} & -\sin \phi_{k} \cos \theta_{k} \\
0 & \sin \theta_{k}
\end{array}\right]\left[\begin{array}{c}
\sin \gamma_{k} e^{j \eta_{k}} \\
\cos \gamma_{k}
\end{array}\right] \\
& =\mathbf{\Xi}_{k} \zeta_{k}
\end{aligned}
$$

where $\theta_{k} \in[0, \pi), \phi_{k} \in(0,2 \pi], \gamma_{k} \in[0, \pi / 2), \eta_{k} \in[-\pi, \pi)$ denotes the $k$ th source's elevation measured from positive vertical $z$-axis, azimuth, auxiliary polarization angle, and polarization phase difference, respectively. The $(\cdot)^{T}$ stands for the transpose. Throughout this work, we assume that different sources have different polarization states.

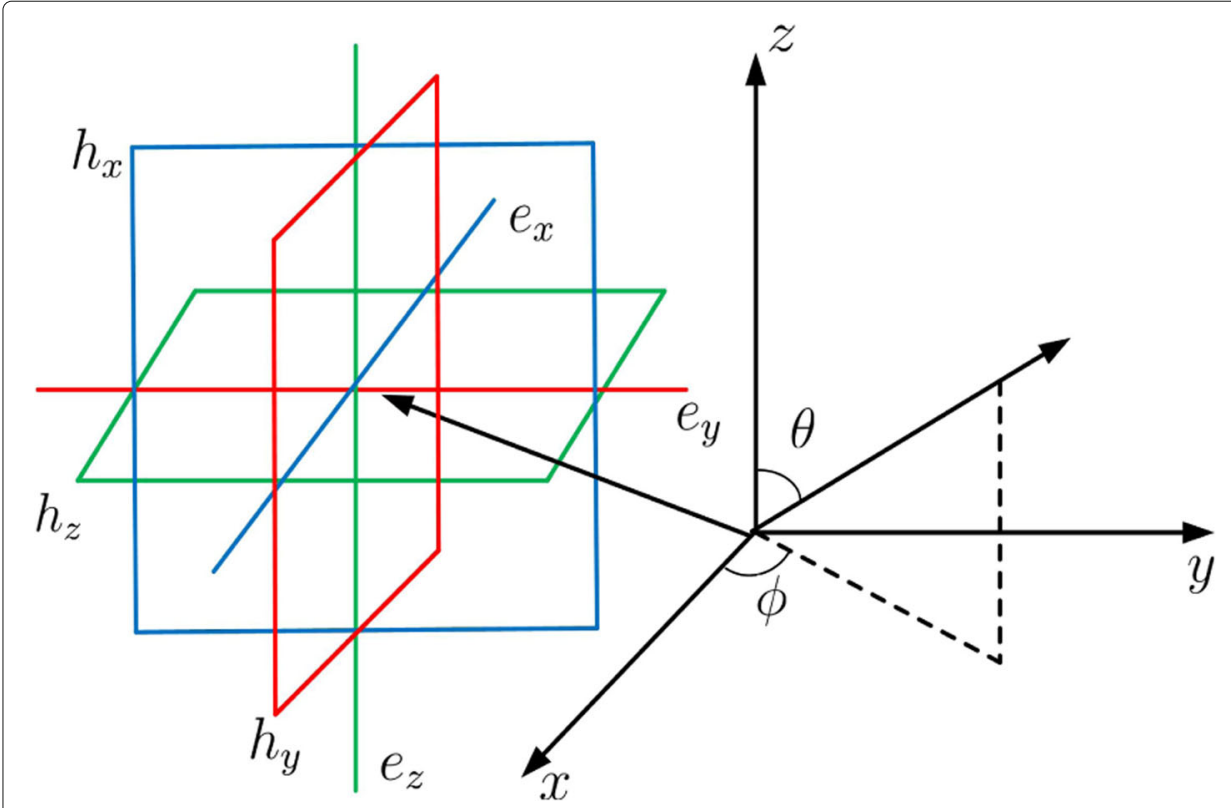

Fig. 1 Diagram of a six-component EMVS 
Then, the normalized Poynting vector $\mathbf{g}_{k}$ is given by

$$
\begin{aligned}
\mathbf{g}_{k} & \triangleq \frac{\mathbf{e}_{k}}{\left\|\mathbf{e}_{k}\right\|} \times \frac{\mathbf{h}_{k}^{*}}{\left\|\mathbf{h}_{k}\right\|} \\
& \triangleq\left[\begin{array}{c}
\mu_{k} \\
v_{k} \\
\omega_{k}
\end{array}\right]=\left[\begin{array}{cc}
\sin \theta_{k} & \cos \phi_{k} \\
\sin \theta_{k} & \sin \phi_{k} \\
\cos \theta_{k}
\end{array}\right]
\end{aligned}
$$

where $(\cdot)^{*}, \times$, and $\|\cdot\|$ represent the complex conjugation, Cartesian product, and $\ell_{2}$ norm, respectively. We use $\mu_{k}, v_{k}, \omega_{k}$ for the direction-cosine functions along the $x$-, $y$-, and $z$-axis, respectively.

In this paper, we consider a typical two-level nested array which is composed of two concatenated ULAs with different inner EMVS spacings. The EMVS linear array is placed along the $y$-axis with a total of $M$ EMVSs as illustrated in Fig. 2. The small ULA has $M_{1}$ sensors with a half-wavelength spacing, whereas the large one has a total of $M_{2}$ sensors with an intersensor spacing of $\left(M_{1}+1\right) \frac{\lambda}{2}$. Thus, the positions of EMVSs in the nested array are given as

$$
\mathbf{z}=\frac{\lambda}{2}\left[1,2, \ldots, M_{1}, M_{1}+1,2 M_{1}+2, \ldots, M_{2}\left(M_{1}+1\right)\right]^{T} .
$$

According to [24], the DOFs for a nested array with identical scalar-sensors could be determined as

$$
\bar{M}=\left\{\begin{array}{l}
\frac{M^{2}-2}{2}+M \text { if } M \text { is even } \\
\frac{M^{2}-1}{2}+M \text { if } M \text { is odd. }
\end{array}\right.
$$

Assume that there are $K$ narrowband far-field completed polarized signals impinging on this array. As a result, the spatial steering vector of the nested EMVS array for the $k$ th source is given by

$$
\mathbf{a}\left(\theta_{k}\right) \triangleq\left[e^{j \frac{2 \pi}{\lambda} z_{1} \sin \theta_{k}}, e^{j \frac{2 \pi}{\lambda} z_{2} \sin \theta_{k}}, \ldots, e^{j \frac{2 \pi}{\lambda} z_{M} \sin \theta_{k}}\right]^{T}
$$

where $\lambda$ denotes the wavelength of the sources and $z_{k}$ denotes the $k$ th element of $\mathbf{z}$. Note that (8) only stands for the spatial relationship among all $M$ EMVSs in the array without taking the physical property of EMVS into account. The $t$ th sample vector of whole array gives as

$$
\begin{aligned}
\mathbf{y}(t) & =\sum_{k=1}^{K} s_{k}(t)\left(\mathbf{a}\left(\theta_{k}\right) \odot \mathbf{p}_{k}\right)+\mathbf{n}(t) \\
& =(\mathbf{A} \odot \mathbf{P}) \mathbf{s}(t)+\mathbf{n}(t) \\
& =\mathbf{A}_{\mathrm{p}} \mathbf{s}(t)+\mathbf{n}(t)
\end{aligned}
$$

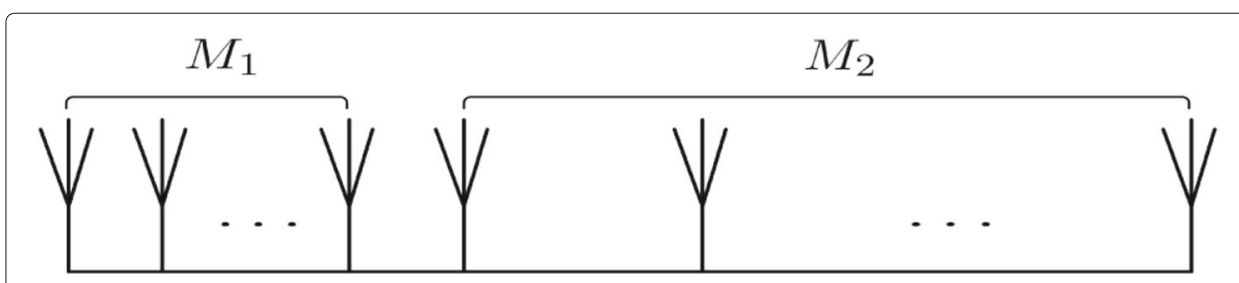

Fig. 2 A nested linear array consists of two concatenated ULAs with $M_{1}$ and $M_{2}$ EMVSs, respectively 
where $\mathbf{A}_{\mathrm{p}} \in \mathbb{C}^{6 M \times K}$ represents the steering matrix of the whole nested EMVS array where $\mathbf{s}(t)$ and $\mathbf{n}(t)$ represent $K$ signals' waveforms at time $t$ and the additive temporally and spatially white Gaussian noise, respectively.

$$
\begin{aligned}
\mathbf{a} \triangleq\left[\mathbf{a}\left(\theta_{1}\right), \ldots, \mathbf{a}\left(\theta_{K}\right)\right] \\
\mathbf{P} \triangleq\left[\mathbf{p}_{1}, \ldots, \mathbf{p}_{K}\right]
\end{aligned}
$$

In this work, we assume that the sources are uncorrelated stationary white Gaussian process. Meanwhile, the additive noise obeys independent and identically distributed (IID) Gaussian distribution, i.e., $n(t) \sim C N\left(0, \sigma_{n}^{2} \mathbf{I}\right)$ with $\sigma_{n}^{2}$ being the noise variance. Furthermore, the signal and noise are uncorrelated. The covariance matrix is calculated as

$$
\begin{aligned}
\mathbf{R} & =\mathbb{E}\left[\mathbf{y}(t) \mathbf{y}^{H}(t)\right] \\
& =\mathbf{a}_{p} \mathbb{E}\left[\mathbf{s}(t) \mathbf{s}^{H}(t)\right] \mathbf{a}_{p}^{H}+\mathbb{E}\left[\mathbf{n}(t) \mathbf{n}^{H}(t)\right] \\
& =\mathbf{a}_{p} \mathbf{D} \mathbf{a}_{p}^{H}+\sigma_{n}^{2} \mathbf{I}_{6 M}
\end{aligned}
$$

where $\mathbf{D}$ denotes the source covariance matrix, $\mathbb{E}[\cdot]$ represents the mathematical expectation. Since the sources are statistically uncorrelated, we have

$$
\mathbf{D}=\operatorname{diag}(\mathbf{d})
$$

where $\mathbf{d}=\left[\sigma_{1}^{2}, \sigma_{2}^{2}, \ldots, \sigma_{K}^{2}\right]^{T}$ with $\sigma_{k}^{2}$ being the power of the $k$ th source and $\operatorname{diag}(\cdot)$ denotes the diagonalization operator that forms an $K \times 1$ vector into a $K \times K$ square matrix with elements on its main diagonal and zeroes elsewhere. Note that the covariance matrix cannot be exactly obtained since the number of samples is finite. Instead, we use the sample covariance matrix (SCM) as

$$
\hat{\mathbf{R}}=\frac{1}{T} \sum_{t=1}^{T} \mathbf{y}(t) \mathbf{y}^{H}(t) .
$$

For simplicity, we use the covariance matrix for derivation of the proposed method. However, it should be kept in mind that the SCM is different from the covariance matrix. In consequence, the signal covariance matrix $\mathbf{R}_{s}$ will not have a perfect diagonal structure. This phenomenon may degrade the performance of the proposed method as we will see in the simulations later.

By vectorizing the covariance matrix, an aperture-enlarged array is obtained, and its observation is given as

$$
\begin{aligned}
\operatorname{vec}(\mathbf{R}) & =\left(\mathbf{a}_{p}^{*} \odot \mathbf{a}_{p}\right) \mathbf{d}+\sigma_{n}^{2} \mathbf{1} \\
& =\left(\mathbf{a}^{*} \odot \mathbf{P}^{*} \odot \mathbf{a} \odot \mathbf{P}\right) \mathbf{d}+\sigma_{n}^{2} \mathbf{1}
\end{aligned}
$$

where $\mathbf{1}=\operatorname{vec}\left(\mathbf{I}_{6 M}\right)$. Note that the steering matrix $\mathbf{a}_{p}^{*} \odot \mathbf{a}_{p} \in \mathbb{C}^{36 M^{2} \times K}$ has a larger size, which extends the array aperture. However, the sources $\mathbf{d}$ become coherent since $\mathbf{s}(t)$ are stationary process. To circumvent this problem, several rank restoration methods, e.g., spatial-smoothing technique and compressive sensing-based approaches, have been suggested. These strategies might not be appropriate for the situation of the nested EMVS array. This is because the aperture-extended array does not have the ULA structure, which is prohibiting the application of spatial-smoothing technique. Note that the spatial matrix $\mathbf{a}$ and polarization matrix $\mathbf{P}$ are alternating arranged as depicted in (18), which are not decoupled. As a matter of fact, the EMVS array can be described as a multi-dimensional 
model. This thereby motivates us to establish the tensor model for the observations of the EMVS array.

\section{Tensor modeling}

\subsection{Han's tensor modeling approach}

The measurement matrix of the whole EMVS array at $t$ th sample is obtained through matrizating (9) as

$$
\begin{aligned}
\mathbf{Y}(t) & =\operatorname{mat}(\mathbf{y}(t)) \\
& =\sum_{k=1}^{K} s_{k}(t)\left(\mathbf{a}\left(\theta_{k}\right) \circ \mathbf{p}_{k}\right)+\mathbf{N}(t) \\
& =\mathcal{A} \times \times_{3} \mathbf{s}(t)+\mathbf{N}(t)
\end{aligned}
$$

where mat(.) denotes the matrization operator, $\mathbf{Y}(t) \in \mathbb{C}^{M \times 6}$, and $\mathcal{A} \in \mathbb{C}^{M \times 6 \times K}$ represents the steering tensor as shown in Fig. 3.

The 3-mode matrix unfolding of the steering tensor is equivalent to the steering matrix of EMVS as

$$
\mathcal{A}_{(3)}^{T}=\mathbf{a}_{p} .
$$

Based on [30], the fourth-dimensional covariance tensor $\mathcal{R}$ is constructed as

$$
\mathcal{R}=\mathbb{E}\left[\mathbf{Y} \circ \mathbf{Y}^{*}\right] \in \mathbb{C}^{M \times 6 \times M \times 6} .
$$

We could regard (23) as a tensor extension of the covariance matrix. To further investigate the structure of $\mathcal{R}$, each element in $\mathcal{R}$ could be expressed as

$$
r_{i_{1} i_{2} i_{3} i_{4}}=\mathbb{E}\left[\mathbf{Y}_{i_{1} i_{2}}(t) \mathbf{Y}_{i_{3} i_{4}}^{*}(t)\right] .
$$

To be specific, we have

$$
\mathcal{R}=\mathbb{E}\left[\left(\mathcal{A} \times{ }_{3} \mathbf{s}+\mathbf{N}\right) \circ\left(\mathcal{A} \times{ }_{3} \mathbf{s}+\mathbf{N}\right)^{*}\right] .
$$

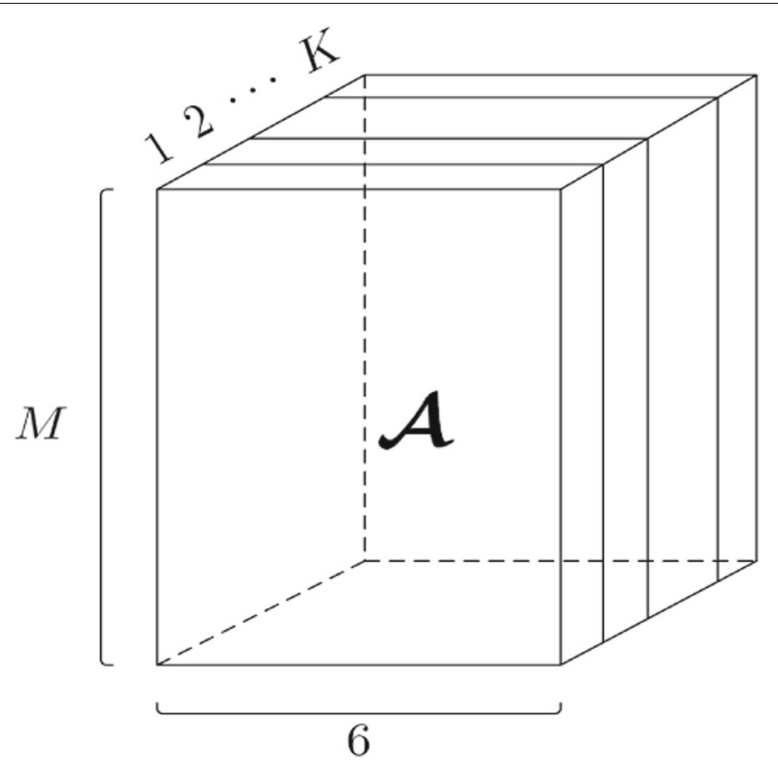

Fig. 3 The steering tensor of a six-component EMVS 
Then perform 2-mode matrization of $\mathcal{R}$, yielding

$$
\begin{aligned}
\mathbf{R}_{\text {ten }} & =\mathcal{R}_{(2)}^{T} \\
& =\mathcal{A}_{\text {nest }} \times_{3} \mathbf{d}+\sigma_{n}^{2} \mathbf{E}_{n}
\end{aligned}
$$

where $\mathcal{A}_{\text {nest }} \in \mathbb{C}^{6 M^{2} \times 6 \times K}$ stands for the steering tensor and

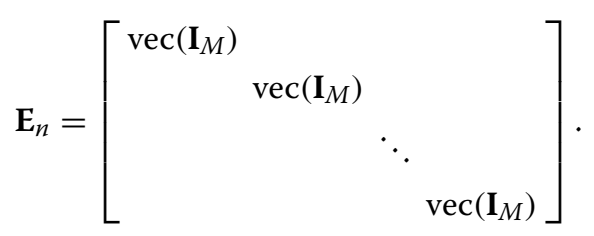

Comparing with (17), (26) has a similar form but with a nested steering tensor of size $6 M^{2} \times 6 \times K$. Taking one horizontal slice of (30) since the DOA and polarization state are coupled in the steering tensor as

$$
\mathcal{A}_{\text {nest }}=\left[\mathcal{A}_{\text {nest }}^{1}, \ldots, \mathcal{A}_{\text {nest }}^{6}\right]
$$

where $\mathcal{A}_{\text {nest }}^{l} \in \mathbb{C}^{6 M^{2} \times K}, l=1, \ldots, 6$ represents the sub-nested steering tensor associated with the $l$ th polarization state in $\mathbf{p}_{k}$. The polarization state and the spatial structure are coupled in the first dimension of the $l$ th sub-nested steering tensor, and it has the following form

$$
\begin{aligned}
\mathcal{A}_{\text {nest }}^{l} & =\mathcal{A}_{(3)}^{H} \odot \mathcal{A}_{l} \\
& =\mathbf{a}_{p}^{*} \odot \mathbf{a}_{l}
\end{aligned}
$$

and $\mathbf{a}_{l}$ denotes one slice of the nested steering tensor associated with $l$ th polarization state

$$
\mathbf{a}_{l}=\left[\begin{array}{ccc}
p_{1 l} e^{-j r_{1} \pi \sin \theta_{1}} & \ldots & p_{K l} e^{-j r_{1} \pi \sin \theta_{K}} \\
p_{1 l} e^{-j r_{2} \pi \sin \theta_{1}} & \ldots & p_{K l} e^{-j d_{2} \pi \sin \theta_{K}} \\
\vdots & \ddots & \vdots \\
p_{1 l} e^{-j r_{M} \pi \sin \theta_{1}} & \ldots & p_{K l} e^{-j r_{M} \pi \sin \theta_{K}}
\end{array}\right] .
$$

where $\mathbf{a}_{l} \in \mathbb{C}^{M \times K}, l=1,2, \ldots, 6$. In a word, the tensor modeling of nested EMVS array could be regarded as folding one of the polarization dimension of the matrix-based steering matrix into a higher dimension. Note that the spatial and polarization dimensions of the nested steering tensor are still coupled.

\subsection{Proposed tensor modeling method}

Different from the existing tensor modeling methods in [30] and [31], we introduce the property of tensor permutation to establish a tensor model of the nested EMVS array.

Definition 4 (Tensor permutation) Consider an N-dimensional tensor, a permutation operator of this tensor is denoted as $\pi=\left[\pi_{1}, \pi_{2}, \ldots, \pi_{N}\right]$, where $\pi_{n} \in\{1,2, \ldots, N\}, n=$ $1,2, \ldots, N$. An $N$-dimensional tensor $\mathcal{C} \in \mathbb{C}^{I_{1} \times I_{2} \ldots \times I_{N}}$ with indexes $i_{1}, i_{2}, \ldots, i_{N}$ after permutation leads to $\mathcal{C}_{\pi} \in \mathbb{C}^{I_{\pi_{1}} \times I_{\pi_{2}} \ldots \times I_{\pi_{N}}}$

$$
\pi:\left(i_{1}, i_{2}, \ldots, i_{N}\right) \mapsto\left(i_{\pi_{1}}, i_{\pi_{2}}, \ldots, i_{\pi_{N}}\right)
$$

For example, we define a permutation as $\pi=[3,2,1]$. Then, a three-dimensional tensor $\mathcal{C} \in \mathbb{C}^{I_{1} \times I_{2} \times I_{3}}$ after permutation is $\mathcal{C}_{\pi} \in \mathbb{C}^{I_{3} \times I_{2} \times I_{1}}$ as shown in Fig. 4 . 

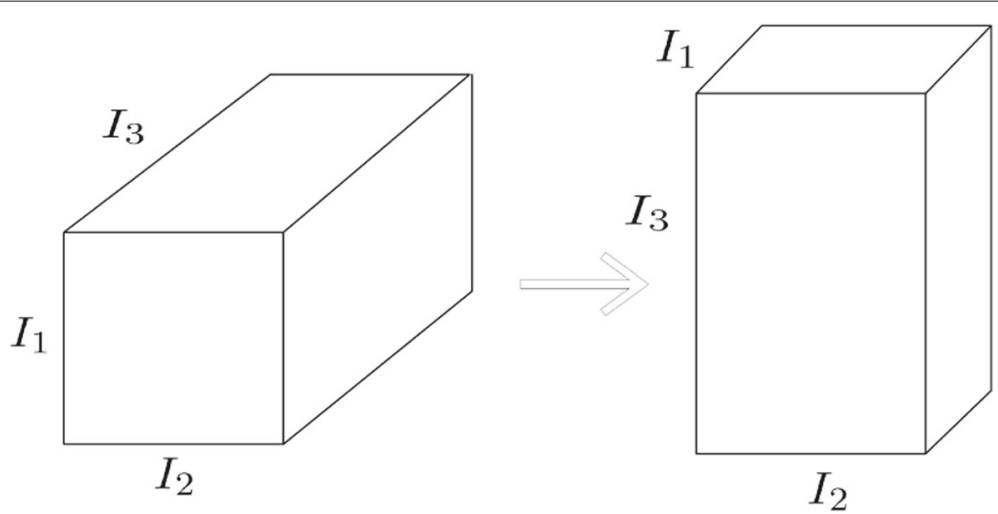

Fig. 4 Diagram of a three-dimensional tensor permutation

Property 2 If an $N$-dimensional tensor $\mathcal{C}^{I_{1} \times \cdots \times I_{N}}$ could be expressed as a outer-product of $N$ vectors, we have the following expression after permutation $\pi=\left[\pi_{1}, \pi_{2}, \ldots, \pi_{N}\right]$ as

$$
\mathcal{C}_{\pi}=\mathbf{c}_{\pi_{1}} \circ \mathbf{c}_{\pi_{2}} \circ \ldots \circ \mathbf{c}_{\pi_{N}}
$$

This property is proved through using Property 1 and the definition of tensor permutation. It represents a special case of rank-one tensors, which is based the fact that tensor permutation will maintain the inter-relationship of each vectors.

Recalling (17), we obtain

$$
\begin{aligned}
\operatorname{vec}(\mathbf{R}) & =\left(\mathbf{a}^{*} \odot \mathbf{P}^{*} \odot \mathbf{a} \odot \mathbf{P}\right) \mathbf{d}+\sigma_{n}^{2} \mathbf{1} \\
& =\sum_{k=1}^{K} \sigma_{k}^{2} \mathbf{a}_{k}^{*} \odot \mathbf{p}_{k}^{*} \odot \mathbf{a}_{k} \odot \mathbf{p}_{k}+\sigma_{n}^{2} \mathbf{1} .
\end{aligned}
$$

According to Property 1, we formulate (33) into a four-dimensional tensor as

$$
\mathcal{G}_{\text {nest }}=\sum_{k=1}^{K} \sigma_{k}^{2} \mathbf{a}^{*}\left(\theta_{k}\right) \circ \mathbf{p}_{k}^{*} \circ \mathbf{a}\left(\theta_{k}\right) \circ \mathbf{p}_{k}+\mathcal{N}_{\text {nest }}
$$

where $\mathcal{G}_{\text {nest }} \in \mathbb{C}^{M \times 6 \times M \times 6}$. Then, by setting $\pi:(1,3,2,4), \mathcal{G}_{\text {nest }}$ after permutation $\pi$ gives

$$
\mathcal{G}_{\pi}=\sum_{k=1}^{K} \sigma_{k}^{2} \mathbf{a}^{*}\left(\theta_{k}\right) \circ \mathbf{a}\left(\theta_{k}\right) \circ \mathbf{p}_{k}^{*} \circ \mathbf{p}_{k}+\mathcal{N}_{\pi} .
$$

Performing 1-mode unfolding of $\mathcal{G}_{\pi} \in \mathbb{C}^{M \times M \times 6 \times 6}$ into a three-dimensional tensor yields

$$
\mathcal{G}_{\pi}^{(1)}=\sum_{k=1}^{K} \sigma_{k}^{2}\left(\mathbf{a}^{*}\left(\theta_{k}\right) \odot \mathbf{a}\left(\theta_{k}\right)\right) \circ \mathbf{p}_{k}^{*} \circ \mathbf{p}_{k}+\mathcal{N}_{\pi}
$$

where $\mathcal{G}_{\pi}^{(1)} \in \mathbb{C}^{M^{2} \times 6 \times 6}$. The spatial and polarization vectors are arranged in order instead of placing alternatively as in (33). Until this step, a three-dimensional tensor with decoupled spatial and polarization factors is constructed. However, $\mathbf{a}^{*}\left(\theta_{k}\right) \odot \mathbf{a}\left(\theta_{k}\right)$ does not obey the Vandermonde structure and incurs several repeated spatial phase factors as pointed out in [30]. Here, we directly remove the repeated ones to reduce the dimension of $\mathbf{a}^{*}\left(\theta_{k}\right) \odot \mathbf{a}\left(\theta_{k}\right)$ while keeping the DOFs unchanged. The selecting and permutation 
matrices are defined as $\mathbf{J}_{s}$ and $\mathbf{J}_{p}$, respectively. Then, we operate them on $\mathcal{G}_{\pi}$ to remove the repeated and arrange the spatial phase factors in order as

$$
\begin{aligned}
\mathcal{G} & =\mathcal{G}_{\pi}^{(1)} \times_{1} \mathbf{J}_{s} \times_{1} \mathbf{J}_{p} \\
& =\sum_{k=1}^{K} \sigma_{k}^{2} \mathbf{b}\left(\theta_{k}\right) \circ \mathbf{p}_{k}^{*} \circ \mathbf{p}_{k}+\mathcal{N}
\end{aligned}
$$

where $\mathcal{G} \in \mathbb{C}^{\bar{M} \times 6 \times 6}$ and

$$
\mathbf{b}\left(\theta_{k}\right)=\left[e^{-j \pi \frac{\bar{M}-1}{2} \sin \theta_{k}}, \ldots, 1, \ldots, e^{j \pi \frac{\bar{M}-1}{2} \sin \theta_{k}}\right]^{T} .
$$

Note that the deleting and selecting matrices are determined by the spatial structure of the nested array and thus can be calculated offline once.

To be specific, each element in $\mathcal{G}$ is expressed as

$$
g_{i_{1} i_{2} i_{3}}=\sum_{k=1}^{K} \sigma_{k}^{2} b_{i_{1} k} p_{i_{2} k}^{*} p_{i_{3} k}+\sigma_{n}^{2} \delta_{i_{1} i_{2} i_{3}}
$$

where $i_{1}=1, \ldots, \bar{M}, i_{2}=1, \ldots, 6, i_{3}=1, \ldots, 6$ and $\delta$ stands for the Dirac function such that

$$
\delta_{i_{1} i_{2} i_{3}}=\left\{\begin{array}{ll}
1 & i_{1}=\frac{\bar{M}+1}{2} \\
0 & \text { elsewhere }
\end{array} \text { and } i_{2}=i_{3} .\right.
$$

Since we have already known the positions of noise items as given in (41), the noise could be easily eliminated from $\mathcal{G}$ to further improve the signal-to-noise ratio (SNR) in real world applications. Performing SVD to (16), the singular value matrix is given as $\Sigma=$ $\operatorname{diag}\left(\hat{\sigma}_{1}^{2}, \ldots, \hat{\sigma}_{6 M}^{2}\right)$. The singular values are decreasingly ordered as $\hat{\sigma}_{1}^{2} \geq \cdots \geq \hat{\sigma}_{K}^{2}>$ $\hat{\sigma}_{K+1}^{2}=\ldots=\hat{\sigma}_{6 M}^{2}$. Thus, an estimate of the noise power could be given as

$$
\hat{\sigma}_{n}^{2}=\frac{1}{6 M-K} \sum_{k=K+1}^{6 M} \hat{\sigma}_{k}^{2} .
$$

It should be emphasized that although the proposed tensor model has a similar form as that in [31], the physical properties behind the model are quite different. In our proposed model, the matrix factors represent spatial and polarization states while dropping the temporal information. Note that the powers of $K$ sources are trivial parameters which will not influence the estimate of parameters.

\section{DOA and polarization estimation}

\subsection{Elevation estimation}

In this subsection, elevation estimation is firstly achieved. Then, we use the results to obtain azimuth and polarization estimates in the next subsection. The proposed tensor modeling approach constructs a three-dimensional tensor which is able to exploit the spatially correlation structure inherent in the EMVS array data. To this end, we use $\mathbf{B}$, $\mathbf{P}$, and $\mathbf{P}^{*}$, respectively, to stand for matrix factors. Thus, we could use CPD to estimate these factors directly. The CPD of $\mathcal{G}$ could achieve the estimates of $\mathbf{B}$ and $\mathbf{P}$ up to permutation and scaling [14]. The Alternating Least Squares (ALS) algorithm is usually applied to conduct the general CPD without a prior knowledge of the structure of matrix factors. It is worth noting that since $\mathbf{B}$ obeys the Vandermonde structure, this problem at hand 
could be solved through Vandermonde constrained CPD (VCPD) [17], which has more a relaxed uniqueness condition.

The noiseless matrix form of $\mathcal{G}$ could be obtained as 1-mode unfolding, which is given by

$$
\mathbf{G}=\mathbf{B D H}^{T}
$$

where

$$
\begin{aligned}
\mathbf{B} & =\left[\mathbf{b}\left(\theta_{1}\right), \mathbf{b}\left(\theta_{2}\right), \ldots, \mathbf{b}\left(\theta_{K}\right)\right] \\
\mathbf{H} & =\mathbf{P}^{*} \odot \mathbf{P} .
\end{aligned}
$$

Consider (43), we divide $\mathbf{G}$ into $L$ overlapped submatrices, each of them has size $M_{s} \times 36$ to obtain the spatial-smoothing of $\mathbf{G}$ along the spatial dimension. Firstly, we define the $l$ th selection matrix as

$$
\mathbf{J}_{l}=\left[\mathbf{0}_{M_{s} \times(l-1)} \mathbf{I}_{M_{s}} \mathbf{0}_{M_{s} \times(L-l)}\right] . \quad 1 \leq l \leq L .
$$

The augmented covariance $\mathbf{G}_{s}$ is then constructed as

$$
\begin{aligned}
\mathbf{G}_{s} & =\left[\mathbf{J}_{1} \mathbf{G}, \ldots, \mathbf{J}_{L} \mathbf{G}\right] \in \mathbb{C}^{M_{s} \times 36 L} \\
& =\mathbf{B}_{s}\left[1, \boldsymbol{\Phi}, \ldots, \boldsymbol{\Phi}^{L-1}\right] \mathbf{D} \mathbf{H}^{T}
\end{aligned}
$$

where $M_{s}=\bar{M}-L+1$ and

$$
\begin{aligned}
\boldsymbol{\Phi} & \triangleq \operatorname{diag}\left(e^{-j \pi \sin \theta_{1}}, \ldots, e^{-j \pi \sin \theta_{K}}\right) \\
\mathbf{B}_{s} & =\left[\mathbf{I}_{M_{s}} \mathbf{0}_{M_{s} \times \bar{M}-M_{s}}\right] \mathbf{B} .
\end{aligned}
$$

Through the constuction of augmented covariance matrix, we restore the rank of $\mathbf{G}$ to achieve a better estimate of the signal subspace. Performing SVD to $\mathbf{G}_{s}$, a low-rank approximation is obtained as

$$
\operatorname{SVD}\left(\mathbf{G}_{s}\right)=\left[\begin{array}{ll}
\hat{\mathbf{U}}_{s} & \hat{\mathbf{U}}_{n}
\end{array}\right]\left[\begin{array}{cc}
\hat{\boldsymbol{\Sigma}}_{s} & \mathbf{0} \\
\mathbf{0} & \hat{\boldsymbol{\Sigma}}_{n}
\end{array}\right]\left[\begin{array}{ll}
\hat{\mathbf{V}}_{s} & \hat{\mathbf{V}}_{n}
\end{array}\right]^{H} .
$$

It follows from (50) and (51) that

$$
\hat{\mathbf{U}}_{s}=\hat{\mathbf{B}}_{s} \mathbf{T}
$$

where $\mathbf{T}$ represents a full-rank matrix. To proceed, we need to define the selection matrices $\mathbf{J}_{s 1}$ and $\mathbf{J}_{s 2}$, which are defined as

$$
\begin{aligned}
& \mathbf{J}_{s 1} \triangleq\left[\begin{array}{ll}
\mathbf{I}_{M_{s}-1} & \mathbf{0}_{\left(M_{s}-1\right) \times 1}
\end{array}\right] \\
& \mathbf{J}_{s 2} \triangleq\left[\begin{array}{ll}
\mathbf{0}_{\left(M_{s}-1\right) \times 1} & \mathbf{I}_{M_{s}-1}
\end{array}\right] .
\end{aligned}
$$

Recall that the steering matrix $\mathbf{B}_{s}$ obeys Vandermonde structure, which indicates the steering matrix has spatial invariance property. The elevation is estimated by applying (52) and (53)

$$
\mathbf{J}_{s 1} \hat{\mathbf{U}}_{s} \boldsymbol{\Phi}=\mathbf{J}_{s 2} \hat{\mathbf{U}}_{s} .
$$

However, there exists subspace perturbation in both handsides. Here, we use the total least squares (TLS) solution to eliminate the influence of subspace perturbation as follows

$$
\left(\mathbf{J}_{s 1} \mathbf{U}_{s}+\Delta \mathbf{U}_{s 1}\right) \Phi=\mathbf{J}_{s 2} \mathbf{U}_{s}+\Delta \mathbf{U}_{s 2}
$$


where $\Delta \mathbf{U}_{s 1}$ and $\Delta \mathbf{U}_{s 2}$ represent the perturbation of signal subspace of both handsides. Note that the left and right handsides of (55) are highly structured and share many elements, which in turn means that they share the same noises. In order to suppress the noise shared in the sub-arrays, the structured least squares ESPRIT (SLS-ESPRIT) is tailored for enhancing the performance of elevation finding. In particular, defining define $\mathbf{U}_{s}^{\mathrm{SLS}}=\mathbf{U}_{s}+\Delta \mathbf{U}_{s}$ and the residual matrix as $\mathbf{F}\left(\mathbf{U}_{s}^{\mathrm{SLS}}, \Phi\right)=\mathbf{J}_{s 1} \mathbf{U}_{s}^{\mathrm{SLS}} \Phi-\mathbf{J}_{s 2} \mathbf{U}_{s}^{\mathrm{SLS}}$ as the residual matrix, the SLS-ESPRIT method attempts to minimize

$$
\min _{\mathbf{U}_{s}, \Phi}\left\|\left[\begin{array}{c}
\mathbf{F}\left(\mathbf{U}_{s}^{\mathrm{SLS}}, \Phi\right) \\
\gamma \Delta \mathbf{U}_{s}
\end{array}\right]\right\|
$$

where $\gamma$ is the weighting factor which is used to avoid trivial solutions. Note that we use the iteration method to solve (56). The $r$ th iteration step is formulated as

$$
\begin{aligned}
\mathbf{F}( & \left(\mathbf{U}_{s, r+1}^{\mathrm{SLS}}, \Phi_{r+1}\right) \\
= & \mathbf{F}\left(\mathbf{U}_{s, r}^{\mathrm{SLS}}+\Delta \mathbf{U}_{s, r}^{\mathrm{SLS}}, \Phi_{r}+\Delta \Phi_{r}\right) \\
= & \left.\mathbf{J}_{s 1}\left(\mathbf{U}_{s, r}^{\mathrm{SLS}}+\Delta \mathbf{U}_{s, r}^{\mathrm{SLS}}\right)\left(\Phi_{r}+\Delta \Phi_{r}\right)\right) \\
& -\mathbf{J}_{s 2}\left(\mathbf{U}_{s, r}^{\mathrm{SLS}}+\Delta \mathbf{U}_{s, r}^{\mathrm{SLS}}\right) \\
= & \mathbf{F}\left(\mathbf{U}_{s, r}^{\mathrm{SLS}}, \Phi_{r}\right) \\
+ & \mathbf{J}_{s 1}\left(\mathbf{U}_{s, r}^{\mathrm{SLS}} \Delta \Phi_{r}+\Delta \mathbf{U}_{s, r}^{\mathrm{SLS}} \Phi_{r}\right)-\mathbf{J}_{s 2} \Delta \mathbf{U}_{s, r}^{\mathrm{SLS}} .
\end{aligned}
$$

Neglecting the second-order term of (58), we have

$$
\min _{\Delta \mathbf{U}_{s, r}^{\mathrm{SLS}}, \Delta \Phi_{r}}\left\|\mathbf{a}_{s}\left[\begin{array}{c}
\Delta \Phi_{r} \\
\Delta \mathbf{U}_{s, r}^{\mathrm{SLS}}
\end{array}\right]+\left[\begin{array}{c}
\mathbf{F}\left(\mathbf{U}_{s, r}^{\mathrm{SLS}}, \Phi_{r}\right) \\
\gamma \Delta \mathbf{U}_{s, r}^{\mathrm{SLS}}
\end{array}\right]\right\|
$$

where

$$
\mathbf{a}_{s}=\left[\begin{array}{cc}
\mathbf{J}_{s 1} \Delta \mathbf{U}_{s, r}^{\mathrm{SLS}} & \Phi_{r} \mathbf{J}_{s 1}-\mathbf{J}_{s 2} \\
\mathbf{0} & \gamma \mathbf{I}
\end{array}\right] .
$$

Thus, the updates of $\Phi_{r}$ and $\mathbf{U}_{s, r}^{\text {SLS }}$ could be expressed in a closed-form, expressed as

$$
\left[\begin{array}{c}
\Delta \Phi_{r} \\
\Delta \mathbf{U}_{s, r}^{\mathrm{SLS}}
\end{array}\right]=-\mathbf{A}_{s}^{\dagger}\left[\begin{array}{c}
\mathbf{F}\left(\mathbf{U}_{s, r}^{\mathrm{SLS}}, \Phi_{r}\right) \\
\gamma \Delta \mathbf{U}_{s, r}^{\mathrm{SLS}}
\end{array}\right]
$$

where $(\cdot)^{\dagger}$ represents the pesudo-inverse operator. After several iterations, the solution of (56) is achieved. Although the proposed SLS-VCPD method is not optimal, it realizes a tradeoff between computational burden and estimation accuracy.

\subsection{Azimuth and polarization joint estimation}

In the above subsection, the estimates of elevations have been determined. This allows us to construct $\mathbf{B}(\hat{\boldsymbol{\theta}})$, leading to the LS estimate of $\mathbf{H}$ as

$$
\hat{\mathbf{H}}=\mathbf{G}^{T}\left(\mathbf{B}(\hat{\boldsymbol{\theta}})^{T}\right)^{\dagger}
$$

where $\hat{\mathbf{H}}$ consists of $K$ vectors, that is 


$$
\hat{\mathbf{H}}=\left[\hat{\mathbf{h}}_{1}, \hat{\mathbf{h}}_{2}, \ldots, \hat{\mathbf{h}}_{K}\right] .
$$

Recalling (45), the $k$ th column of $\mathbf{H}$ is indeed an outer product of polarization vectors. Dividing $\hat{\mathbf{h}}_{k}$ into six equally sized concatenated vectors and stack them along column to form a square matrix, we have

$$
\operatorname{mat}\left(\hat{\mathbf{h}}_{k}\right)=\lambda_{k} \hat{\mathbf{p}}_{k} \circ \hat{\mathbf{p}}_{k}^{*} \in \mathbb{C}^{6 \times 6}
$$

Performing SVD to $\operatorname{mat}\left(\mathbf{h}_{k}\right)$, an approximate estimate of the polarization information vector is obtained as

$$
\hat{\mathbf{p}}_{k}=\hat{\mathbf{u}}_{k}
$$

where $\hat{\mathbf{u}}_{k}$ stands for the singular vector associated with the largest singular value.

We now have achieved an estimate of the polarization vector. According to (4), the estimated Poynting vector of $k$ th source is

$$
\hat{\mathbf{g}}_{k}=\frac{\hat{\mathbf{e}}_{k}}{\left\|\hat{\mathbf{e}}_{k}\right\|} \times \frac{\hat{\mathbf{h}}_{k}^{*}}{\left\|\hat{\mathbf{h}}_{k}\right\|}
$$

It follows that the estimated azimuth is calculated as

$$
\hat{\phi}_{k}=\arctan \left(\hat{v}_{k} / \hat{\mu}_{k}\right)
$$

Substituting $\hat{\boldsymbol{\theta}}_{k}$ and $\hat{\boldsymbol{\phi}}_{k}$ into (4), we achieve the estimate of $\hat{\boldsymbol{\Xi}}_{k}$. The polarization parameters associated with $k$ th signal are hence calculated as

$$
\begin{aligned}
& \hat{\gamma}_{k}=\arctan \frac{\hat{\zeta}_{k 1}}{\hat{\zeta}_{k 2}} \\
& \hat{\eta}_{k}=\angle \hat{\zeta}_{k 1} \quad k=1,2, \ldots, K
\end{aligned}
$$

where

$$
\hat{\zeta}_{k}=\left[\begin{array}{ll}
\hat{\zeta}_{k 1} & \hat{\zeta}_{k 2}
\end{array}\right]^{T}=\hat{\mathbf{\Xi}}_{k}^{\dagger} \hat{\mathbf{p}}_{k} .
$$

Note that DOA and polarization associated with the same source is auto-paired since they share the same eigenvector. Thus, no extra pairing step is needed. Table 1 concludes the DOA and polarization estimation procedure.

\section{Identifiability and CRB}

\subsection{Identifiability}

In this subsection, we first review the sufficient uniqueness condition with EMVS array from the CPD perspective. Note that an $\mathrm{N}$-dimensional tensor is defined as a rank-one tensor if it could be written into a outer product of $N$ vectors. Thus, $\mathcal{G}$ is rank- $K$ since it is the minimal number of $K$ rank-one tensor as given in (38). Consider a three-dimensional

Table 1 Algorithm of Joint Elevation-Azimuth-Polarization Estimation

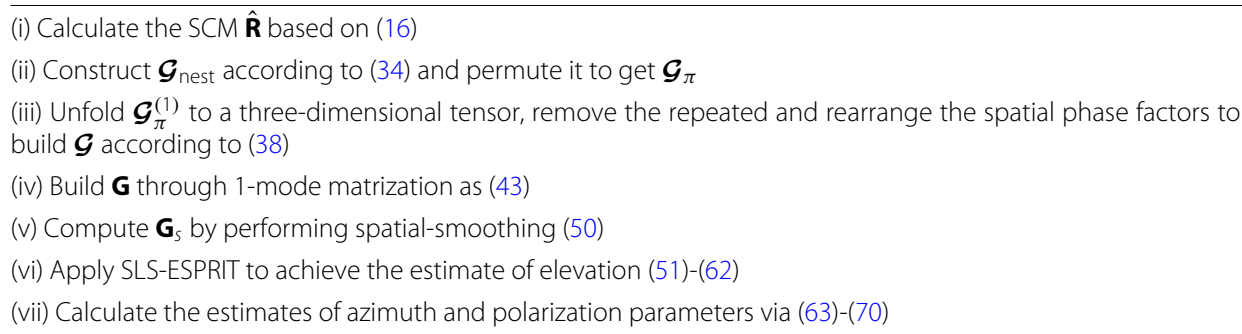


$\mathcal{X} \in \mathbb{C}^{I_{1} \times I_{2} \times I_{3}}$ with rank- $K$ which means that it is a sum of $K$ outer product of three vectors, which could be expressed as

$$
\mathcal{X}=\sum_{k=1}^{K} a_{i_{1} k} b_{i_{2} k} c_{i_{3} k} .
$$

The uniqueness condition of a tensor with CPD relies on KrusKal's condition. The Kruskal-rank, termed as $k r$, is defined as the maximum number of $J$ such that every $J$ columns of a matrix are linearly independent. And it is denoted as $k r(\mathbf{a})=J$. Thus, the KrusKal's condition provides a sufficient uniqueness condition for a three-dimensional tensor. That is, three matrix factors satisfy

$$
k r(\mathbf{a})+k r(\mathbf{B})+k r(\mathbf{C}) \geq 2 K+2
$$

a, $\mathbf{B}$, and $\mathbf{C}$ are unique up to scaling and permutation [14].

For a complete six-components EMVS, its observation data always has $k r(\mathbf{P}) \geq 3$. Thus, for an $M$-elements EMVS ULA, the upper bound for the uniqueness condition when sources are uncorrelated gives

$$
K \leq M+\operatorname{rank}(\mathbf{P})-2 .
$$

Usually, it holds that $\operatorname{rank}(\mathbf{P}) \geq 4$ for arbitrary DOA and polarization parameters. So the maximum number of identifiable sources is

$$
K \leq M+2 .
$$

Comparing with the scalar-sensor ULA, the EMVS array could resolve more sources. Now, we consider the nested EMVS array, the sufficient uniqueness condition with the proposed model (38) yields

$$
k r(\mathbf{B})+k r\left(\mathbf{P}^{*}\right)+k r(\mathbf{P}) \geq 2 K+2 .
$$

Since $\mathbf{B}$ is a Vandermonde matrix, it is straightforward to obtain $k r(\mathbf{B})=\bar{M}$. Also, the manifold of a six-component EMVS is free of rank-2 ambiguity, which indicates that $k r(\mathbf{P}) \geq 3$ [32]. Substituting $k r(\mathbf{P})=3$ into (76) to obtain the upper bound of identifiability with CPD yields

$$
K \leq \frac{\bar{M}}{2}+2 \text {. }
$$

Comparing with (75), the nested EMVS array obviously provides more DOFs. However, the uniqueness condition could be further relaxed if we take the Vandermonde structure of $\mathbf{B}$ and ESPRIT method together into consideration. According to the results in [17], we could directly obtain the upper bound of identifiability after applying spatial smoothing as

$$
K \leq \min (6(\bar{M}-L+1), 6 L) .
$$

It should be noted that the proposed method perhaps cannot resolve the maximum number of sources provided by the upper bound since there exists errors between the SCM and covariance matrix when the number of samples is finite, and SNR is not sufficient high. For more discussions about this phenomenon, readers can refer to [27].

\subsection{Underdetermined CRB}

The stochastic CRB with EMVSs array model has been well discussed in literature. However, the existing CRB could not be directly applied to the underdetermined case in which 
the number of sources excesses the number of EMVSs. Under the assumption that $\mathbf{R}_{s}$ is a diagonal matrix, we extend the stochastic CRB for underdetermined DOA estimation with scalar-sensor array to the EMVSs array cases. Indeed, the derived CRB generalizes the existing results so that the scalar-sensor array could be regarded as a special case of the EMVS array. For simplicity, the variance of noise is assumed to be known. The parameter vector composed by DOAs, polarization, and source powers is defined as

$$
\zeta=\left[\boldsymbol{\theta}^{T}, \boldsymbol{\phi}^{T}, \boldsymbol{\gamma}^{T}, \boldsymbol{\eta}^{T}, \mathbf{d}\right]^{T}
$$

According to [33], the $(m, n)$ th element of the Fisher information matrix (FIM) is given by

$$
\mathrm{FIM}_{m n}=T \operatorname{tr}\left[\frac{\partial \mathbf{R}}{\partial \zeta_{m}} \mathbf{R}^{-1} \frac{\partial \mathbf{R}}{\partial \zeta_{n}} \mathbf{R}^{-1}\right]
$$

where $\operatorname{tr}(\cdot)$ represents the trace operator. Since $\operatorname{tr}(\mathbf{a B})=\operatorname{vec}\left(\mathbf{a}^{T}\right)^{T} \operatorname{vec}(\mathbf{B})$ and $\operatorname{vec}(\mathbf{a X B})=$ $\left(\mathbf{B}^{T} \otimes \mathbf{a}\right) \operatorname{vec}(\mathbf{X})$, we have

$$
\mathrm{FIM}_{m n}=T \operatorname{tr}\left[\frac{\partial \mathbf{r}}{\partial \zeta_{m}}\right]^{H}\left(\mathbf{R}^{-T} \otimes \mathbf{R}^{-1}\right)\left[\frac{\partial \mathbf{r}}{\partial \zeta_{n}}\right]
$$

where $\mathbf{r} \triangleq \operatorname{vec}(\mathbf{R}), \otimes$ denotes the Kronecker product. The first-order derivatives of $\mathbf{r}$ with respect to each element of $\zeta$ is defined as

$$
\begin{aligned}
\frac{\partial \mathbf{r}}{\partial \boldsymbol{\zeta}}= & {\left[\frac{\partial \mathbf{r}}{\partial \theta_{1}}, \ldots, \frac{\partial \mathbf{r}}{\partial \theta_{K}}, \frac{\partial \mathbf{r}}{\partial \phi_{1}}, \ldots, \frac{\partial \mathbf{r}}{\partial \phi_{K}},\right.} \\
& \left.\frac{\partial \mathbf{r}}{\partial \gamma_{1}}, \ldots, \frac{\partial \mathbf{r}}{\partial \gamma_{K}}, \frac{\partial \mathbf{r}}{\partial \eta_{1}}, \ldots, \frac{\partial \mathbf{r}}{\partial \eta_{K}}, \frac{\partial \mathbf{r}}{\partial p_{1}^{2}}, \ldots, \frac{\partial \mathbf{r}}{\partial p_{K}^{2}}\right] .
\end{aligned}
$$

According to (33), it is straightforward to get

$$
\frac{\partial \mathbf{r}}{\partial \zeta}=\left[\mathbf{a}_{\text {nest }}^{(1)}\left(\mathbf{I}_{4} \otimes \mathbf{P}\right), \mathbf{a}_{n e s t}\right]
$$

where $\mathbf{a}_{\text {nest }}^{(1)}=\mathbf{a}_{p}^{(1)^{*}} \odot \mathbf{a}_{p}+\mathbf{a}_{p}^{*} \odot \mathbf{a}_{p}^{(1)}$ and

$$
\begin{gathered}
\mathbf{a}_{p}^{(1)}=\left[\frac{\partial \mathbf{a}_{p}}{\partial \theta_{1}}, \ldots, \frac{\partial \mathbf{a}_{p}}{\partial \theta_{K}}, \frac{\partial \mathbf{a}_{p}}{\partial \phi_{1}}, \ldots, \frac{\partial \mathbf{a}_{p}}{\partial \phi_{K}},\right. \\
\left.\frac{\partial \mathbf{a}_{p}}{\partial \gamma_{1}}, \ldots, \frac{\partial \mathbf{a}_{p}}{\partial \gamma_{K}}, \frac{\partial \mathbf{a}_{p}}{\partial \eta_{1}}, \ldots, \frac{\partial \mathbf{a}_{p}}{\partial \eta_{K}}\right] .
\end{gathered}
$$

To proceed, we need to define the following matrices

$$
\begin{aligned}
& \mathbf{W}=\left(\mathbf{R}^{-T} \otimes \mathbf{R}^{-1}\right)^{1 / 2} \\
& \mathbf{Q}_{1}=\mathbf{W} \mathbf{a}_{\text {nest }}^{(1)}\left(\mathbf{I}_{4} \otimes \mathbf{P}\right) \\
& \mathbf{Q}_{2}=\mathbf{W} \mathbf{a}_{\text {nest }} .
\end{aligned}
$$

Then, the FIM could be expressed into a compact form as

$$
\mathrm{FIM}=T\left[\begin{array}{ll}
\mathbf{Q}_{1}^{H} \mathbf{Q}_{1} & \mathbf{Q}_{1}^{H} \mathbf{Q}_{2} \\
\mathbf{Q}_{2}^{H} \mathbf{Q}_{1} & \mathbf{Q}_{2}^{H} \mathbf{Q}_{2} .
\end{array}\right]
$$

The underdetermined stochastic CRB of $\boldsymbol{\theta}, \boldsymbol{\phi}, \boldsymbol{\gamma}, \boldsymbol{\eta}$ with nested EMVS array is calculated as

$$
\mathrm{CRB}=\frac{1}{T} \operatorname{binv}\left(\mathbf{Q}_{1}^{H} \Pi_{\mathbf{Q}_{2}}^{\perp} \mathbf{Q}_{1}\right)
$$


where $\Pi_{\mathbf{Q}_{2}}^{\perp}=\mathbf{I}-\mathbf{Q}_{2}\left(\mathbf{Q}_{2}^{H} \mathbf{Q}_{2}\right)^{-1} \mathbf{Q}_{2}^{H}$, and binv(.) stands for blockwise inverse operator which takes inverse of the block matrices with size $K \times K$ on the main block diagonal of (87).

\section{Simulation results and discussions}

This section gives various numerical examples to show the performance of the proposed SLS-VCPD method. First, we evaluate root mean square error (RMSE) performance of our proposed method for elevation, azimuth, and polarization estimations. Second, we compare the proposed approach with the state-of-the-art tensor-based schemes which use the SLS-VCPD technique and EMVS array. Throughout all simulations, a nested EMVS array with $M=6$ EMVSs is adopted, which is depicted in Fig. 2. The position of these EMVSs are placed on $\mathbf{z}=\frac{\lambda}{2}[1,2,3,4,8,12]^{T}$.

The RMSE of elevation estimation is defined as

$$
\operatorname{RMSE}_{\theta}=\sqrt{\mathbb{E}\left\{\frac{1}{K} \sum_{k=1}^{K}\left(\hat{\theta}_{k}-\theta_{k}\right)^{2}\right\}} .
$$

Note that RMSEs of azimuth and polarization are computed by the same formula, but the variables are different. In addition, the SNR is defined as $10 \log _{10}\left(\left\|\mathbf{A}_{p} \mathbf{S}\right\|_{F}^{2} /\|\mathbf{N}\|_{F}^{2}\right)$ where $\|\cdot\|_{F}$ represents the Frobenius norm.

\subsection{Performance analysis}

In Figs. 5 and 6, we present the RMSE performances of elevations, azimuth, and polarization, respectively. The parameters of two sources are set as $\boldsymbol{\theta}=\left[30^{\circ}, 40^{\circ}\right], \phi=\left[30^{\circ}, 60^{\circ}\right]$, $\gamma=\left[20^{\circ}, 45^{\circ}\right]$, and $\eta=\left[15^{\circ}, 30^{\circ}\right]$, respectively. It is seen that the RMSE performances improve when SNR or the number of samples increases.

In Fig. 7, we study the influence of the number of iterations on the proposed SLS-VCPD method. In this simulation, the parameters of four sources are randomly chosen as $\boldsymbol{\theta}=\left[49.0^{\circ}, 3.8^{\circ}, 44.8^{\circ}, 74.3^{\circ}\right], \boldsymbol{\phi}=\left[103.1^{\circ}, 101.4^{\circ}, 33.2^{\circ}, 67.7^{\circ}\right], \gamma=$ $\left[14.7^{\circ}, 46.9^{\circ}, 65.2^{\circ}, 33.7^{\circ}\right]$, and $\eta=\left[-67.0^{\circ},-101.7^{\circ},-114.2^{\circ}, 30.5^{\circ}\right]$. The number of receive samples is set as $T=100$. The curves of SLS-VCPD with iteration number, i.e., $n=1$ and $n=3$, are also plotted for comparison. We observe that the proposed SLSVCPD method outperforms the LS-VCPD scheme as expected. Also, the performance of SLS-VCPD remains unchanged as the number of iterations increases, which indicates that the proposed SLS-VCPD method has convergenced within one iteration.

\subsection{Overdetermined case}

In this subsection, we focus on the elevation estimation performance in the overdetermined case. To examine the performance of the nested EMVS array, two array configurations are considered, i.e., ULA and nested array. The number of elements in the EMVS ULA is set as $M=6$ with $K=2$ impinging sources. We compare CPD with EMVS ULA, namely, ULA-CPD [18], tensor-MUSIC [30], SS-CPD [31], and proposed SLS-VCPD methods using the nested EMVS array. Note that the tensor-MUSIC, SS-CPD, and SLS-VCPD methods are constructed using nested array, and thereby, we conceal the item "nested array" for simplification. Note that the SLS-VCPD method only uses one iteration step. The number of spatial smoothing is set as $L=2$ for both SS-CPD and SLS-VCPD methods. For comparison, the polarization states of sources are prior known 


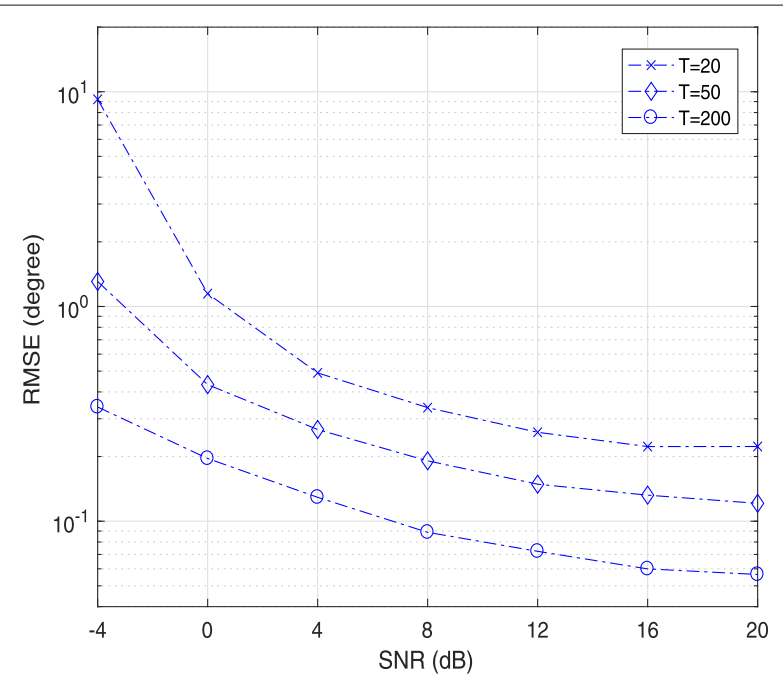

(a) Elevation estimation

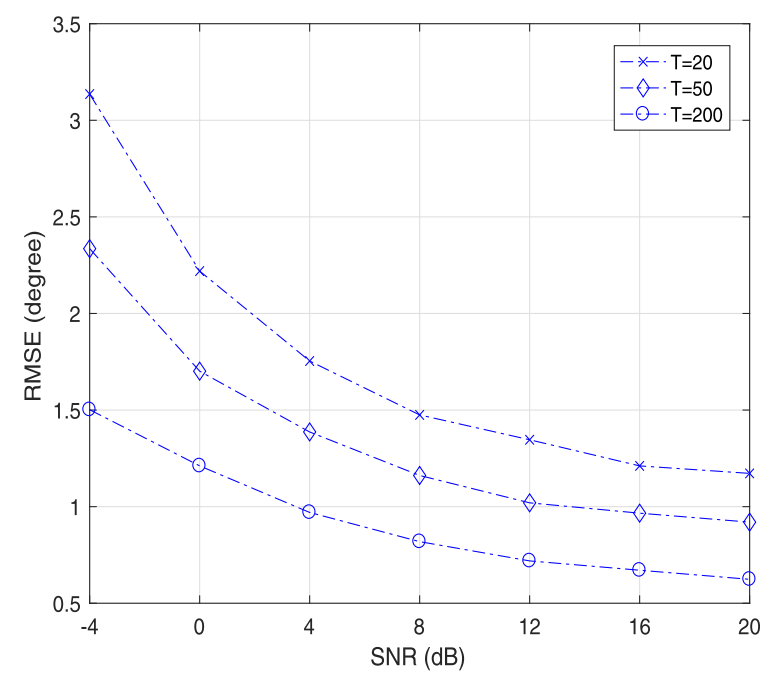

(b) Azimuth estimation

Fig. 5 DOA estimation performance analysis of SLS-VCPD method versus SNR with different number of samples. A nested EMVS array is equipped with $M=6$ sensors. The number of sources is set as $K=2$

to the tensor-MUSIC method to reduce the computational burden. Also, the stochastic CRB with nested EMVS array is plotted as a benchmark. The Monte-Carlo trials for each simulation are set as 1000 .

In Fig. 8, we compare the elevation RMSEs of all methods while varying SNR from $-4 \mathrm{~dB}$ to $20 \mathrm{~dB}$ for a fixed $T=50$ samples. The parameters of two sources are the same as those in Fig. 5. It is seen that the SLS-VCPD approach results in the lowest RMSE among the aforementioned schemes. The nested EMVS array offers more DOFs comparing with the EMVS ULA. Thus, we can observe better RMSE performance. However, the RMSEs of parameter estimation methods with nested EMVS array converges to nonzero value since there exists errors according to (17) when the source covariance matrix is not strictly diagonal [27]. In addition, we also give average CPU run time for each method for comparison. The ULA-CPD, tensor-MUSIC, SS-CPD, and SLS-VCPD methods demand 0.23 s, $3.31 \mathrm{~s}$, 


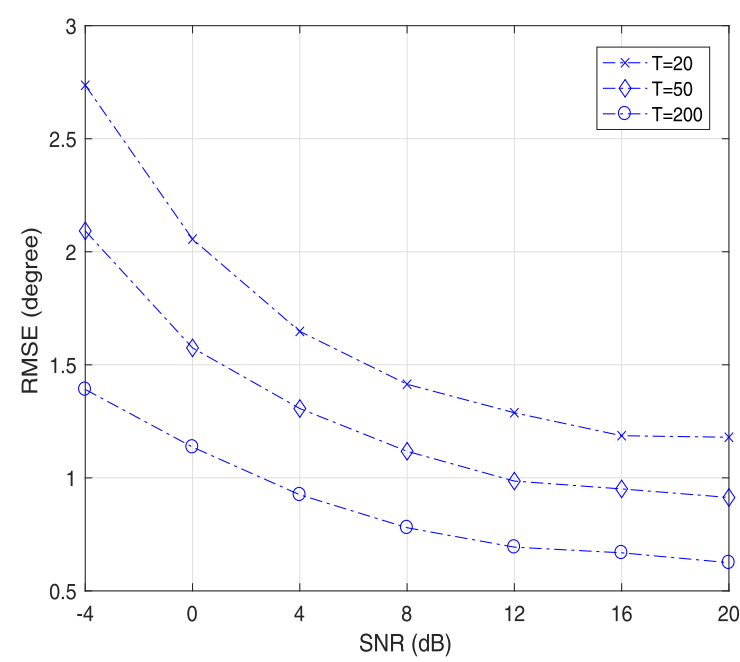

(a) Auxiliary polarization estimation

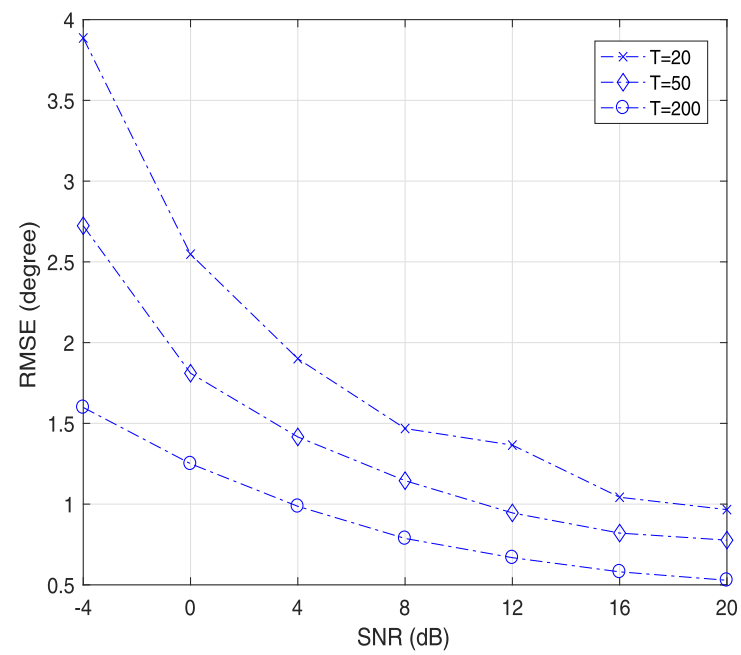

(b) Polarization phase difference estimation

Fig. 6 Polarization estimation performance analysis of SLS-VCPD method versus SNR with different number of samples. A nested EMVS array is equipped with $M=6$ sensors. The number of sources is set as $K=2$

$0.47 \mathrm{~s}$, and $0.51 \mathrm{~s}$, respectively. It could be seen that the proposed SLS-VCPD method has a modest computation complexity among four method. The tensor-MUSIC method requires multi-dimensional searching over the whole parameter space, which needs the most computations.

In Fig. 9, we examine the elevation RMSEs of all methods while varying the number of samples from $T=20$ to $T=200$ with fixed SNR $=10 \mathrm{~dB}$. The parameters of sources remain the same as those in Fig. 5. It can be observed that the SLS-VCPD algorithm is clearly advantageous when compared with the other methods.

In Fig. 10, we compare the elevation resolvabilities of all the above approaches, $T=50$ samples are used and 500 Monte-Carlo trials have been carried out. Two spatially closely sources are assumed to impinge on the array with $\boldsymbol{\theta}=\left[20^{\circ}, 22^{\circ}\right], \boldsymbol{\phi}=\left[30^{\circ}, 30^{\circ}\right], \gamma=$ 


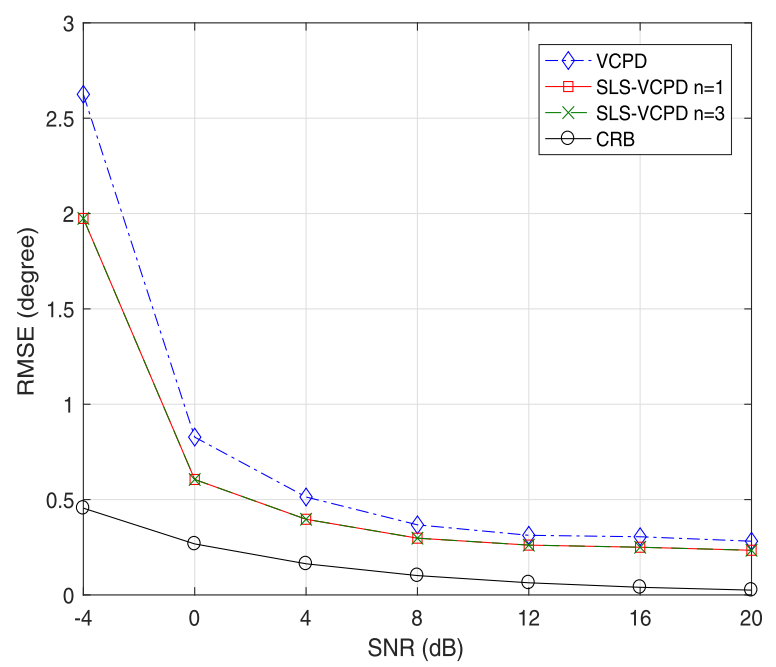

Fig. 7 Performance analysis of the influence of iteration number of the RMSE versus SNR for four signals with $M=6$

$\left[0^{\circ}, 15^{\circ}\right]$, and $\eta=\left[15^{\circ}, 30^{\circ}\right]$. The resolvable condition defined for the elevation estimation is given as [34]

$$
\left|\hat{\theta}_{k}-\theta_{k}\right|<\frac{\left|\theta_{1}-\theta_{2}\right|}{2}
$$

where $\hat{\theta}_{k}, k=1,2$, represents the estimated elevation of two sources. We can see that the SLS-VCPD method provides the best resolvability among all the schemes. SLS-VCPD, ULA-CPD, and SS-CPD methods attain one in terms of probability of successful resolution when SNR $\geq 12 \mathrm{~dB}$. However, the Tensor-MUSIC method fails to provide the reliable detection even though SNR becomes sufficiently large.

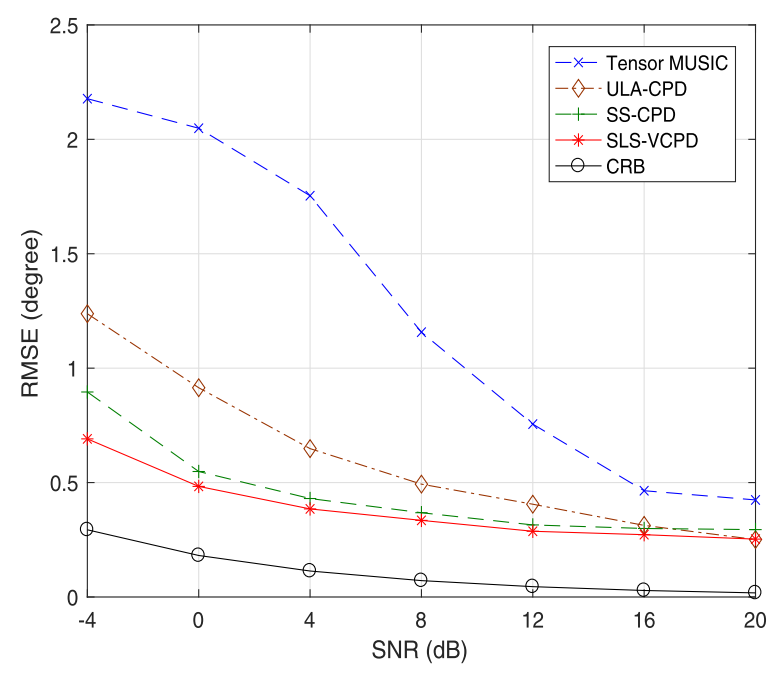

Fig. 8 RMSE versus SNR for two sources with $M=6$ and $T=50$ 


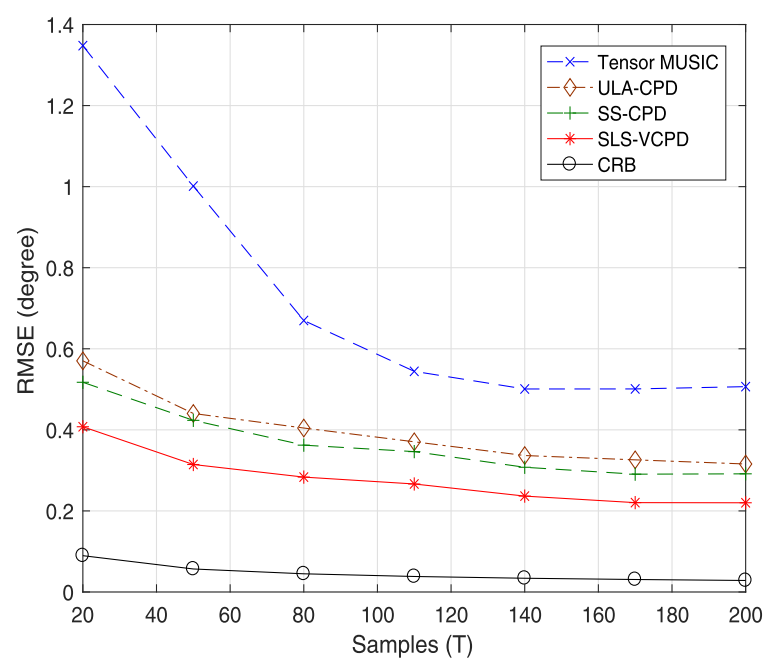

Fig. 9 RMSE versus the number of samples for two sources with $M=6$ and $S N R=10 \mathrm{~dB}$

\subsection{Underdetermined case}

In this subsection, the underdetermined case where the number of sources is more than the number of elements in the original EMVS array. Recall that an $M$-elements EMVS ULA could resolve up to $M+2$ sources. Here, we consider an $M=6$ elements nested EMVS array with $K=10$ impinging sources, which is called the underdetermined case. In this scenario, the ULA-CPD method $[15,18]$ cannot resolve these sources. The parameters of ten sources are randomly chosen. We examine the performance of the ALS-CPD with our proposed model (38), SS-CPD and SLS-VCPD methods based on the nested EMVS array. The tensor-MUSIC method is no longer included since the polarization state is very difficult to be a prior known when the number of sources is so large. Also, the computational burden of tensor-MUSIC method becomes intolerable.

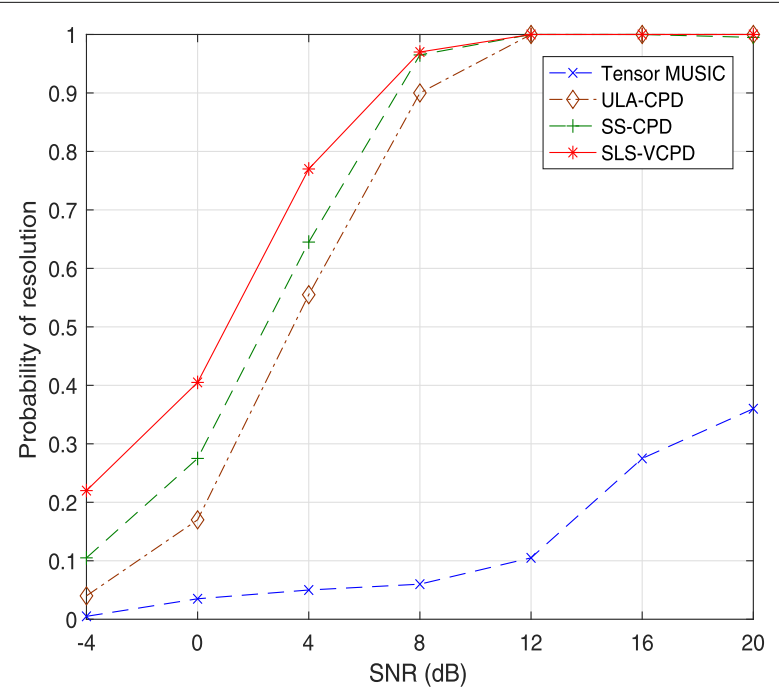

Fig. 10 Resolution ability versus SNR for two spatial closely located sources with $M=6$, and the number of samples is set as $T=50$ 


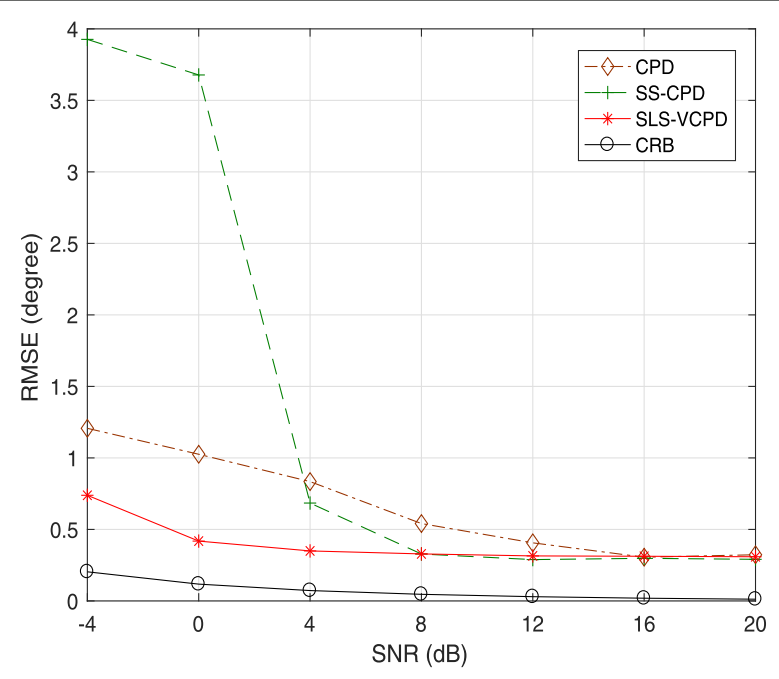

Fig. 11 RMSE versus SNR for ten sources with $M=6$ and $T=2000$

Figure 11 shows the elevation RMSEs of three methods versus SNR for $T=2000$ samples. It is obvious that the SLS-VCPD method offers the lowest RMSE especially when SNR $<8 \mathrm{~dB}$. When SNR $>16 \mathrm{~dB}$, the ALS-CPD, SS-CPD, and SLS-CPD schemes have almost the same RMSE performance. The RMSE curves saturate as SNR is sufficiently high. This phenomenon is caused by the approximation of (17) since the SCM cannot be strictly diagonal when the number of samples is finite as we discussed in the overdetermined case.

In Fig. 12, we study the elevation RMSEs versus the number of samples with $S N R=10 \mathrm{~dB}$. The proposed approach outperforms the other two algorithms when $T<1600$. Moreover, it is observed that the SLS-VCPD and SS-CPD methods result in the almost same performance when the number of samples is greater than 1600 .

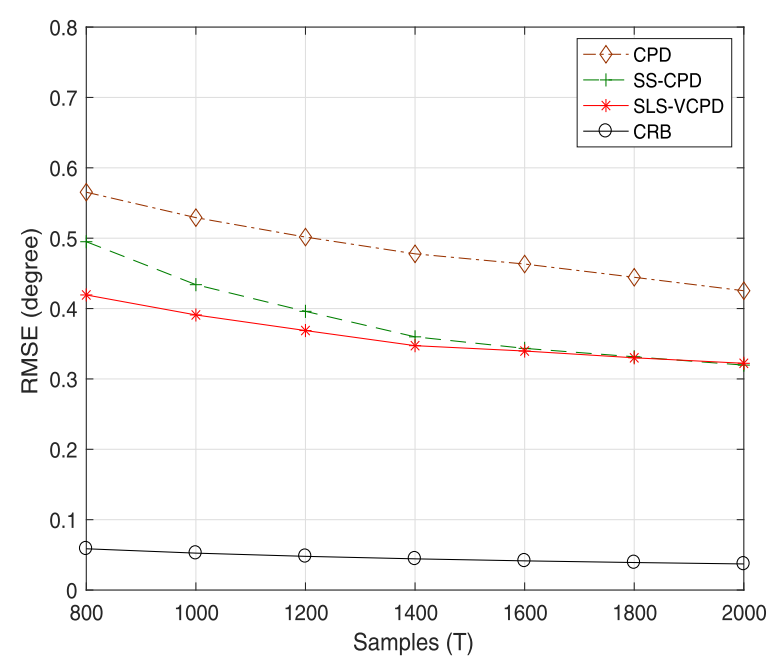

Fig. 12 RMSE versus the number of samples for ten sources with $M=6$ and $T=2000$ 


\title{
7 Methods
}

A tensor modeling method for elevation, azimuth, and polarization estimation with nested electromagnetic vector-sensor arrays is proposed. Since the signals are uncorrelated, we build the SCM into a CP model through tensor permutation. The spatial and polarization information are separated. Then, the SLS-VCPD method is implemented on this model to calculate the elevation and azimuth. Next, the polarization is estimated based on the structure of the vector-sensor.

\section{Conclusions}

The issue of joint elevation, azimuth, and polarization estimation in underdetermined case with the nested EMVS array has been addressed in this work. This array could be modeled into a high-dimensional tensor. The property of tensor permutation is introduced to separate the spatial and polarization vectors which are originally coupled in the steering matrix. This allows us to develop a tensor model of the nested EMVS array with extended spatial aperture. Furthermore, since the spatial and polarization are decoupled, which enables an efficient computational method for auto-pairing parameter estimation, avoiding exhaustive multi-parameter searching. We also investigate the uniqueness condition offered by the proposed SLS-VCPD approach. Besides, the underdetermined stochastic CRB with nested EMVS array is derived. Numerical examples confirm the superiority of our proposed method.

\begin{abstract}
Abbreviations
ALS: Alternating least squares; CRB: Cramér-Rao bound; CPD: Canonical polyadic decomposition; DOA: Direction-ofarrival; DOF: Degree-of-freedom; EMVS: Electromagnetic vector-sensor; ESPRIT: Estimation of signal parameters via rotational invariance techniques; HOSVD: Higher-order singular value decomposition; IID: Independent and identically distributed; MUSIC: Multiple signal classification; RMSE: Root mean square error; SCM: Sample covariance matrix; SLS: Structured least square; SNR: Signal-to-noise ratio; ULA: Uniform linear array; VCPD: Vandermonde constrained CPD
\end{abstract}

\section{Authors' contributions}

MYC proposed the method and wrote the manuscript. XPM advised on the method and checked the simulations. LH revised the manuscript. The authors read and approved the final manuscript.

Funding

This work was supported by the Key Program of National Natural Science Foundation of China $(61831009$, U1713217).

Availability of data and materials

Data sharing not applicable to this article as no datasets were generated or analyzed during the current study.

Competing interests

The authors declare that they have no competing interests.

\section{Author details}

${ }^{1}$ School of Electronics and Information Engineering, Harbin Institute of Technology, Xidazhi Street, 150001 Harbin, China. ${ }^{2}$ Key Laboratory of Marine Environmental Monitoring and Information Processing, Ministry of Industry and Information Technology, Xidazhi Street, 150001 Harbin, China. ${ }^{3}$ College of Information Engineering, Shenzhen University, Nanhai Road, 518060 Shenzhen, China.

Received: 25 April 2019 Accepted: 6 July 2020

Published online: 27 July 2020

\section{References}

1. S. Chintagunta, P. Ponnusamy, 2D-DOD and 2D-DOA estimation using the electromagnetic vector sensors. Signal Process. 147, 163-172 (2018)

2. H. Hou, X. Mao, Y. Liu, Oblique projection for direction-of-arrival estimation of hybrid completely polarised and partially polarised signals with arbitrary polarimetric array configuration. IET Signal Process. 11, 893-900 (2017)

3. C. C. Ko, J. Zhang, A. Nehorai, Separation and tracking of multiple broadband sources with one electromagnetic vector sensor. IEEE Trans. Aerosp. Electron. Syst. 38(3), 1109-1116 (2002)

4. J. F. Bull, Field probe for measuring vector components of an electromagnetic field. U.S. Patent 5300885A (1994)

5. G. F. Hatke, Performance analysis of the SuperCART antenna array. MIT Lincoln Lab., Cambridge, MA, Proj. Rep. AST-22 (1992) 
6. A. Nehorai, E. Paldi, Vector-sensor array processing for electromagnetic source localization. IEEE Trans. Signal Process. 42(2), 376-398 (1994)

7. J. Li, P. Stoica, Efficient parameter estimation of partially polarized electromagnetic waves. IEEE Trans. Signal Process. 42(11), 3114-3125 (1994)

8. D. Rahamim, J. Tabrikian, R. Shavit, Source localization using vector sensor array in a multipath environment. IEEE Trans. Signal Process. 52(11), 3096-3103 (2004)

9. S. Miron, N. Le Bihan, J. Mars, Quaternion-MUSIC for vector-sensor array processing. IEEE Trans. Signal Process. 54(4), 1218-1229 (2006)

10. X. Yuan, Estimating the DOA and the polarization of a polynomial- phase signal using a single polarized vector-sensor. IEEE Trans. Signal Process. 60(3), 1270-1282 (2012)

11. Q. Cheng, Y. Hua, Performance analysis of the MUSIC and PENCIL-MUSIC algorithms for diversely polarized array. IEEE Trans. Signal Process. 42(11), 3150-3165 (1994)

12. M. D. Zoltowski, K. T. Wong, ESPRIT-based 2-D direction finding with a sparse uniform array of electromagnetic vector sensors. IEEE Trans. Signal Process. 48(8), 2195-2204 (2000)

13. K. T. Wong, M. D. Zoltowski, Uni-vector-sensor ESPRIT for multisource azimuth, elevation, and polarization estimation. IEEE Trans. Antennas Propag. 45(10), 1467-1474 (1997)

14. N. D. Sidiropoulos, R. Bro, G. B. Giannakis, Parallel factor analysis in sensor array processing. IEEE Trans. Signal Process. 48(8), 2377-2388 (2000)

15. X. Guo, S. Miron, D. Brie, S. Zhu, X. Liao, A CANDECOMP/PARAFAC perspective on uniqueness of DOA estimation using a vector sensor array. IEEE Trans. Signal Process. 59(7), 3475-3481 (2011)

16. M. Sørensen, L. De Lathauwer, Multiple invariance ESPRIT for nonuniform linear arrays: a coupled canonical polyadic decomposition approach. IEEE Trans. Signal Process. 64(4), 3693-3704 (2016)

17. M. Sørensen, L. De Lathauwer, Blind signal separation via tensor decomposition with Vandermonde factor: canonical polyadic decomposition. IEEE Trans. Signal Process. 61(22), 5507-5519 (2013)

18. S. Miron, Y. Song, D. Brie, K. Wong, Multilinear direction finding for sensor-array with multiple scales of invariance. IEEE Trans. Aerosp. Electron. Syst. 51(3), 2057-2070 (2015)

19. M. Yang, J. Ding, B. Chen, X. Yuan, A multiscale sparse array of spatially spread electromagnetic-vector-sensors for direction finding and polarization estimation. IEEE access. 6, 9807-9818 (2018)

20. L. de Lathauwer, B. de Moor, J. Vanderwalle, A multilinear singular value decomposition. SIAM J. Matrix Anal. Appl. 21(4), 1253-1278 (2000)

21. F. Roemer, M. Haardt, G. Del Galdo, Analytical performance assessment of multi-dimensional matrix-and tensor-based ESPRIT-type algorithms. IEEE Trans. Signal Process. 62(10), 2611-2625 (2014)

22. X. Gong, Z. Liu, Y. Xu, M. L. Ahmad, Direction-of-arrival estimation via twofold mode-projection. Signal Process. 89(5), 831-842 (2009)

23. P. Forster, G. Ginolhac, M. Boizard, Derivation of the theoretical performance of a Tensor MUSIC algorithm. Signal Process. 129, 97-105 (2016)

24. P. Pal, P. P. Vaidyanathan, Nested array: a novel approach to array processing with enhanced degrees of freedom. IEEE Trans. Signal Process. 58(8), 4167-4181 (2010)

25. P. P. Vaidyanathan, P. Pal, Sparse sensing with co-prime samplers and arrays. IEEE Trans. Signal Process. 59(2), $573-586(2011)$

26. X. Han, T. Shu, J. He, W. Yu, Polarization-angle-frequency estimation with linear nested vector sensors. IEEE Access. 6, 36916-36926 (2018)

27. M. Wang, A. Nehorai, Coarrays, MUSIC, and the Cramér- Rao bound. IEEE Trans. Signal Process. 65(4), 933-946 (2017)

28. A. Koochakzadeh, P. Pal, Cramér-Rao bounds for underdetermined source localization. IEEE Signal Process. Lett. 23(7), 919-923 (2016)

29. C.-L. Liu, P. P. Vaidyanathan, Cramér-Rao bounds for coprime and other sparse arrays, which find more sources than sensors. Dig. Signal Process. 61, 43-61 (2017)

30. K. Han, A. Nehorai, Nested vector-sensor array processing via tensor modeling. IEEE Trans. Signal Process. 62(10), 2542-2553 (2014)

31. R. Wei, D. Li, J. Zhang, A novel PARAFAC model for processing the nested vector-sensor array. Sensors. 18(11), 3708 (2018)

32. K.-C. Tan, K.-C. Ho, A. Nehorai, Linear independence of steering vectors of an electromagnetic vector sensor. IEEE Trans. Signal Process. 44(12), 3099-3107 (1996)

33. B. Ottersten, P. Stoica, R. Roy, Covariance matching estimation techniques for array signal processing applications. Dig. Signal Process. 8(3), 185-210 (1998)

34. H. L. Van Trees, In Optimum Array Processing. (Wiley, New York, 2002)

\section{Publisher's Note}

Springer Nature remains neutral with regard to jurisdictional claims in published maps and institutional affiliations. 